\title{
Solitary Endobronchial Papilloma Mimicking a Neoplastic Lesion
}

\author{
Smadhi Hanen ${ }^{1,2,3}{ }^{*}$, Khalfallah Ikbel ${ }^{1,2,3}$, Diouani Mohamed Fethi ${ }^{4}$, Abdennadher Mahdi ${ }^{5}$, \\ Attia Monia ${ }^{6}$, Ayadi-Kaddour Aida7 ${ }^{7}$ Megdiche Mohamed Lamine ${ }^{1,2,3}$ \\ ${ }^{1}$ Department of Pneumology Ibn Nafis, Abderrahman Mami University Hospital, Ariana, Tunisia \\ ${ }^{2}$ Faculté de Médecine de Tunis, Université Tunis El Manar, Tunis, Tunisia \\ ${ }^{3}$ Faculté des Sciences de Bizerte, Université de Carthage, Carthage, Tunisia \\ ${ }^{4}$ Laboratoire d'Epidémiologie et de Microbiologie Vétérinaire Institut Pasteur de Tunis, Tunis, Tunisia \\ ${ }^{5}$ Department of Thoracic Surgery, Abderrahman Mami University Hospital, Ariana, Tunisia \\ ${ }^{6}$ Department of Radiology, Abderrahman Mami University Hospital, Ariana, Tunisia \\ ${ }^{7}$ Department of Pathology, Abderrahman Mami University Hospital, Ariana, Tunisia \\ Email: *smadhi_hanen@yahoo.fr
}

How to cite this paper: Hanen, S., Ikbel, K., Fethi, D.M., Mahdi, A., Monia, A., Aida, A.-K. and Lamine, M.M. (2018) Solitary Endobronchial Papilloma Mimicking a Neoplastic Lesion. Open Journal of Respiratory Diseases, 8, 38-42. https://doi.org/10.4236/ojrd.2018.83005

Received: July 2, 2018

Accepted: August 13, 2018

Published: August 16, 2018

Copyright $\odot 2018$ by authors and Scientific Research Publishing Inc. This work is licensed under the Creative Commons Attribution International License (CC BY 4.0).

http://creativecommons.org/licenses/by/4.0/

\section{(c) (i) Open Access}

\begin{abstract}
Papilloma is a rare benign tumor. Laryngeal location is the most frequent. The bronchial involvement remains unusual. It represents $0.38 \%$ of pulmonary tumors. We report the case of a 39-year-old, smoker man, who consulted for a cough following during six months with persistent radiological opacity despite antibiotic treatment. The physical examination was without particularities. Flexible bronchoscopy revealed a swollen endobronchial lesion completely obstructing the orifice of the intermediate trunk. Bronchial biopsies at this level showed inflammatory mucosa. The chest CT scan objectified an endobronchial tissular mass in the intermediate trunk. Pulmonary neoplasia was suspected. Diagnostic and therapeutic surgery was performed. Histopathological examination concluded to an endobronchial squamous papilloma.
\end{abstract}

\section{Keywords \\ Papilloma, Smoking, Lung, Cancer, Surgery}

\section{Introduction}

The papilloma is a rare benign entity [1]. The Solitary respiratory papilloma (SRP) is an uncommon pathologic condition of the lower respiratory tract, with less than 60 cases reported in the literature [2]. It accounts for $0.38 \%$ of all pulmonary tumors. Although the precise etiology of respiratory papillomas remains 
unknown, the general consensus is that the majority are due to infection caused by the human papilloma virus (HPV). If papillomas of the upper airways, frequently referred to as juvenile laryngotracheal papillomatosis, are more frequent in childhood, the SRP are more common in adult population. Papillomas are normally classified into three categories: multiple papillomas, inflammatory polyps, and solitary papillomas [3] [4].

Malignant transformation is rare but has been reported. The rate of occurrence of carcinoma from a papilloma is $7.8 \%$ [5] and is not unusual in patients who smoke or who have received irradiation or chemotherapy [6] [7]. We report a case of solitary endobronchial papilloma mimicking a neoplastic lesion diagnosed by surgical resection.

\section{Reported Case}

A 39-year-old man, smoker, with no past history, was addressed to our institution for exploration of a cough evolving since six months. There was no history of hemoptysis, weight loss, night sweats, or change in appetite. The physical examination revealed a temperature of $37.6^{\circ} \mathrm{C}$, a respiratory rate of 18 breaths/min, a heart rate of 80 beats $/ \mathrm{min}$, a blood pressure of $120 / 70 \mathrm{mmHg}$, and a room air oxygen saturation of $96 \%$. A chest examination was normal. A complete blood count, erythrocyte sedimentation rate, liver function test, and Creactive protein were within normal limits. The chest-X-ray revealed alveolar opacity in the right Fowler. We indicated antibiotic treatment based on the combination of amoxicillin and clavulanic acid for a duration of 14 days. Investigations of tuberculosis infection by direct examination were negative in sputum as well as cultures. The evolution was marked by persistence of cough and the imaging opacity. Hence, neoplastic lesion was suspected since the patient was smoker. The chest CT scan showed an endobronchial tissular mass in the intermediate trunk, an unaerated collapse of the middle lobe with an excavated parenchymatous condensation of the right fowler associated with right mediastinal nodes (Figure 1). Flexible bronchoscopy revealed a swollen endobronchial lesion completely obstructing the orifice of the intermediate trunk (Figure 2). Cytology and bronchial biopsies at this level were negative for malignant cells. But the fear of lung cancer conducted us to indicate a surgical resection. Therefore, the patient was operated on by postero-lateral thoracotomy for diagnostic and therapeutic purposes. He underwent a Right lower lobectomy. Pathologic study of the surgical piece showed a polypoid bronchial mucosa, lined by a hyperplastic, papillomatous and acanthosic epithelium without signs of malignancy suggestive of squamous cell papilloma (Figure 3). The Otorhinolaryngologic examination did not demonstrate associated laryngeal papilloma. Human Papilloma Virus (HPV) serology was negative. The evolution is marked by the clinical improvement of the patient. A follow-up CT scan realized after six months didn't show a recurrence of the tumor.

\section{Discussion}

The solitary pulmonary papilloma category of tumors is relatively unusual. It is 


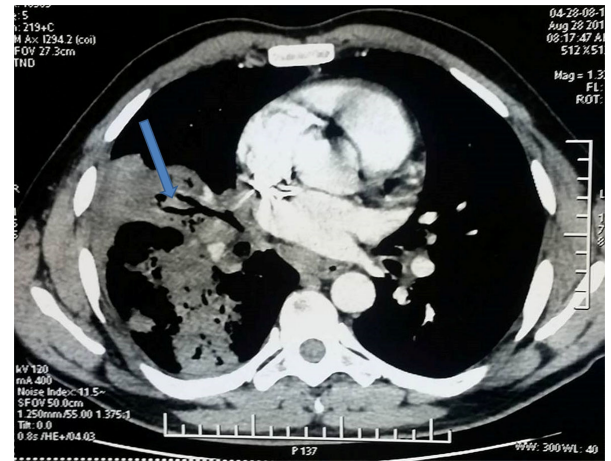

Figure 1. CT Scan showed an endobronchial tissue bud in the intermediate trunk with an excavated parenchymatous condensation of the lower right lobe.

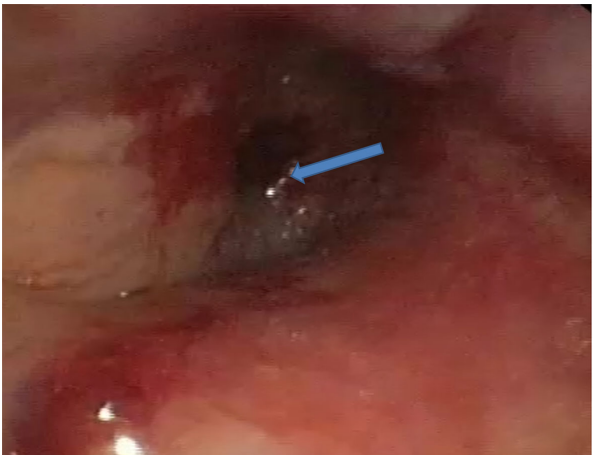

Figure 2. Flexible bronchoscopy revealed a swollen endobronchial lesion completely obstructing the orifice of the intermediate trunk.

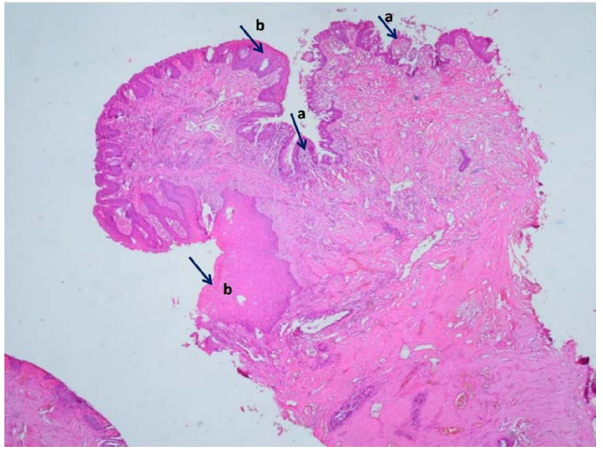

Figure 3. Endobronchial papilloma with polypoid bronchial mucosa (a), lined by a metaplastic, papillomatous and acanthosic epithelium, without signs of malignancy (b) (HEx40).

commonly found in genitalia or oral cavity but it is very unusual to be found in the airways as in our patient. Solitary endobronchial papilloma is a rare form representing only $0.5 \%$ of all types of papilloma and $0.3 \%$ of all lung tumors [1]. Papillomas of the lung are relatively uncommon but it must be considered in the differential diagnosis of any benign or malignant tumor. It is usually classified into three categories: squamous cell papilloma, glandular papilloma, and mixed (squamous cell and glandular papilloma). Malignant transformation is rare. It is a rare pathology that occurs commonly in middle-aged adult with a male pre- 
dominance and a frequent association with smoking. The role of HPV in the genesis of this tumor remains controversial. It would have a role in the pathogenesis of the squamous variant but not in the others [8]. The main complication of this pathology is its malignant transformation, hence the importance of surgical excision. In fact, the rate of occurrence of carcinoma from a papilloma is $7.8 \%$ [5]. In fact, the HPV seems to play a pathogenetic role in some squamous cell papillomas. So, in the benign multiple squamous papillomas of the larynx, trachea, and bronchi, HPV types 6 and 11 were detected using in situ hybridization. Infections with HPV, serotypes 16 or 18, are related with malignant transformation in squamous papillomas [9]. Therefore, the most important apprehension for the pathologist is to distinguish this benign tumor from malignant tumors, principally intraoperatively, since the misdiagnosis of adenocarcinoma, will almost certainly quick unnecessary surgical treatment. Also, it should be noted that the complete lesion should be submitted for lasting histological evaluation to definitively exclude malignant tumor.

The particularity of our case is first, the clinical presentation since it is a central papilloma which comes to clinical attention due to bronchial obstruction, consequent recurrent pneumonia mimicking a neoplastic lesion essentially in smoker patient. In any case, pathologists may more commonly meet such tumors on frozen section. It should be noted that the total lesion should be submitted for lasting histologic evaluation to definitively exclude malignancy. In second time, the location of papilloma is unusual, since it's commonly laryngeal. This endobronchial location can simulate a lung cancer particularly in smoker men.

\section{Conflicts of Interest}

The authors declare no conflicts of interest regarding the publication of this paper.

\section{References}

[1] Tryfon, S., Dramba, V., Zoglopitis, F., Iakovidis, D., Sakkas, L., Kontakiotis, T. and Galanis, N. (2012) Solitary Papillomas of the Lower Airways: Epidemiological, Clinical, and Therapeutic Data during a 22-Year Period and Review of the Literature. Journal of Thoracic Oncology, 7, 643-648. https://doi.org/10.1097/JTO.0b013e3182468d06

[2] Emerson, L.L. and Layfield, L.J. (2012) Solitary Peripheral Pulmonary Papilloma Evaluation on Frozen Section: A Potential Pitfall for the Pathologist. Pathology-Research and Practice, 208, 726-729. https://doi.org/10.1016/j.prp.2012.09.007

[3] Roviaro, G.C., Varoli, F. and Pagnini, C.A. (1981) Is the Solitary Papilloma of the Bronchus Always a Benign Tumor? ORL JOtorhinolaryngol Relat Spec, 43, 301-308. https://doi.org/10.1159/000275550

[4] Spencer, H., Dail, D.H. and Arneaud, J. (1980) Non-Invasive Bronchial Epithelial Papillary Tumors. Cancer, 45, 1486-1497.

https://doi.org/10.1002/1097-0142(19800315)45:6<1486::AID-CNCR2820450632>3. $0 . \mathrm{CO} ; 2-\mathrm{H}$ 
[5] Lie, E.S., Engh, V., Boysen, M., et al. (1994) Squamous Cell Carcinoma of the Respiratory Tract Following Laryngeal Papillomatosis. Acta Oto-Laryngologica, 114, 209-212. https://doi.org/10.3109/00016489409126044

[6] Rabett, W. (1965) Juvenile Laryngeal Papillomatosis, Relation of Irradiation to Malignant Degeneration in This Disease. Annals of Otology, Rhinology \& Laryngology, 74, 1149-1163. https://doi.org/10.1177/000348946507400421

[7] Galloway, T., Soper, G. and Elsen, J. (1960) Carcinoma of the Larynx after Irradiation for Papilloma. Archives of Otolaryngology, 72, 289-294. https://doi.org/10.1001/archotol.1960.00740010297001

[8] Harada, H., Miura, K., Tsutsui, Y., Mineta, H., Urano, M., Abe, M., et al. (2000) Solitary Squamous Cell Papilloma of the Lung in a 40-Year-Old Woman with Recurrent Laryngeal Papillomatosis. Pathology International, 50, 431-439.

https://doi.org/10.1046/j.1440-1827.2000.01055.x

[9] Katial, R.K., Ranlett, R. and Whitlock, W.L. (1994) Human Papilloma Virus Associated with Solitary Squamous Papilloma Complicated by Bronchiectasis and Bronchial Stenosis. Chest, 106, 1887-1889. https://doi.org/10.1378/chest.106.6.1887 